Arq. Bras. Med. Vet. Zootec., v.66, n.2, p.374-380, 2014

\title{
Avaliação da atividade anti-helmíntica de extratos brutos de plantas da Floresta Amazônica e Mata Atlântica brasileira sobre Haemonchus contortus
}

[Evaluation of the anti-helminthic activity of crude extracts from the Brazilian Amazon and Mata Atlântica plants against Haemonchus contortus]

M.P.V. Cunha, A.F. Alves Neto, I.B. Suffredini*, L.J.C. Abel

Universidade Paulista - UNIP - São Paulo, SP

\section{RESUMO}

No mundo todo, as verminoses são causa de considerável prejuízo econômico na criação de ovinos. As perdas estão relacionadas ao retardo na produção, custos com tratamentos profiláticos e, em casos extremos, à morte dos animais. O objetivo deste trabalho foi verificar a ação anti-helmíntica de extratos vegetais sobre nematoides como alternativa terapêutica no tratamento da verminose em ovinos. Extratos orgânicos e aquosos foram obtidos de plantas nativas da Floresta Amazônica e Mata Atlântica, e foram testados na concentração de $100 \mu \mathrm{g} / \mathrm{mL}$ em ensaios de toxicidade in vitro contra ovos de Haemonchus contortus e de $200 \mu \mathrm{g} / \mathrm{mL}$ contra suas larvas. Na concentração estabelecida, apresentaram significante atividade relacionada à inibição de eclosão de ovos; em particular, o extrato orgânico de folhas e frutos de Trichilia sp. e o extrato aquoso dos órgãos aéreos de Phyllanthus attenuatus. Os resultados in vitro sugerem que extratos aquosos e orgânicos dessas plantas podem oferecer novas alternativas de controle da verminose em ovinos a partir de produtos naturais.

Palavras-chave: Haemonchus contortus, anti-helmínticos, Floresta Amazônica, extratos vegetais, controle parasitário

\begin{abstract}
Helminthosis play a crucial role in small ruminant production leading to enormous economic losses particularly in areas where extensive grazing is practiced. It causes a loss of production through mortality, weight loss, reduced milk and wool production. Haemonchus contortus is a highly pathogenic small ruminant helminthes parasite, which is capable of causing acute diseases and high mortality. Therefore, there is an urgent need to seek alternative or complementary solutions to the control of parasitic nematodes of ruminants. The tropical forests concentrate the highest biodiversity in the world, particularly those located in Brazil, whose species richness is estimated to account for $20 \%$ of the total world richness and little is known about the chemical and pharmacological potentialities of Brazilian tropical forests. In this work, organic and aqueous extracts were tested on eggs and in the larval development test on Haemonchus contortus. The organic extract obtained from leaves and fruits of Trichilia sp. and the aqueous extract obtained from the aerial organs of Phyllanthus attenuates showed significant activity related to the inhibition of $\mathrm{H}$. contortus egg eclosion. In vitro results suggest that both extracts, as well as eleven other active extracts, can offer alternatives in sheep worm control.
\end{abstract}

Keywords: Haemonchus contortus, anti-helmintics, Amazon rain forest, plant extracts, parasite control

\section{INTRODUÇÃO}

A criação de ovinos tem desempenhado importante papel econômico na pecuária brasileira. No que diz respeito à participação no mercado mundial, a importação de carne ovina passou de 2,3 mil toneladas em 1992 para 14,7 mil toneladas em 2000, representando um crescimento acima de $600 \%$. A importação de

Recebido em 22 de outubro de 2012

Aceito em 1 de outubro de 2013

*Autor para correspondência (corresponding author)

E-mail: ibsuffredini@yahoo.com.br 
carne caprina também apresentou um crescimento bastante significativo (EMBRAPA CNPC, acessado em 2012).

Dentre os fatores que causam prejuízos na ovino e caprinocultura, as verminoses causadas pelo parasita Haemonchus contortus ocupam lugar de destaque, uma vez que este é o nematódeo de patogenia mais significante para pequenos ruminantes (Jasmer e Mc Guire, 1996) e altamente prevalente no Brasil (Amarante, 2001). As consequências das infecções causadas por esse parasita são o atraso de desenvolvimento corporal dos cordeiros, a queda na produção e na qualidade da carne e da lã (Ramos et al., 2004) e a morte de animais jovens, nos casos mais graves (Molento et al., 2004). Um dos principais fatores ligados à eficiência do ciclo de vida e infecção dos nematoides de ovinos é a resistência a fármacos anti-helmínticos atualmente empregados (Echevarria e Pinheiro, 1989). Por isso o uso de produtos naturais aparece como alternativa terapêutica para o tratamento de verminoses, (Githiori et al., 2006).

O emprego de plantas medicinais tem sido feito há séculos pelos agricultores para o tratamento do parasitismo e melhorar o desempenho produtivo dos animais. No entanto, a evidência científica sobre a eficácia antiparasitária da maioria dos produtos vegetais é limitada, apesar de sua ampla utilização. A validação científica dos efeitos antiparasitários e possíveis efeitos colaterais de produtos vegetais em ruminantes é necessária antes da sua adoção como um novo método para controle de parasitas (Githiori et al., 2006). O Brasil abriga a maior biodiversidade do planeta. Sua biota conhecida está entre 170 e 210 mil espécies de plantas, sendo que as descritas representam apenas 49.520 espécies, embora seja um número crescente (Capobianco, 2001). A Floresta Amazônica e a Mata Atlântica constituem cerca de $30 \%$ dos remanescentes de florestas tropicais existentes sobre a superfície terrestre, abrigando não somente a maior diversidade biológica do planeta (Mittermeier et al., 1999), como também alta variabilidade genética expressa na forma de componentes fitoquímicos (Hamann et al., 1991). Diante desse contexto, verificamos a pertinência de se realizar estudos relacionados ao potencial farmacológico de plantas tropicais brasileiras, especificamente da Mata Atlântica brasileira e da Floresta Amazônica, como agentes anti-helmínticos.
Este trabalho tem como objetivo realizar ensaios de identificação de extratos vegetais de plantas brasileiras que sejam ativos contra ovos e larvas de Haemonchus contortus, através de testes in vitro.

\section{MATERIAIS E MÉTODOS}

Para a obtenção e preparo dos extratos, as plantas foram coletadas em florestas brasileiras, após obtenção de licenças de coleta e acesso aos recursos genéticos emitidas pelo IBAMA e do CGen (MMA) (12A/2008). As espécies foram coletadas em igapó, florestas inundadas pelo Rio Negro. Exemplares de cada espécie (exsicatas) foram registrados e depositados no Herbário UNIP (Suffredini et al., 2007a).

Diferentes órgãos de cada espécie foram coletados (p.ex., órgãos aéreos, folhas ou caule) e foram secos em estufa de circulação de ar (Fanem), a $40^{\circ} \mathrm{C}$. Depois, foram moídos em moinho de facas (Holmes) e submetidos à maceração, por 24 horas, com diclorometano:metanol (1:1) (Merk), seguido de uma segunda maceração, por mais 24 horas, com água destilada Milli-Q (Millipore). Os extratos aquosos foram liofilizados (Virtis) e os orgânicos, rotaevaporados (Buchii), para depois serem mantidos sob congelamento até o uso (Suffredini et al., 2006).

Os extratos orgânicos foram diluídos em dimetilsulfóxido $50 \%$ (DMSO 50\%) e os aquosos, em água. Foram preparados inicialmente a uma concentração de $2 \mathrm{mg} / \mathrm{mL}$. Depois, foram diluídos 10 vezes, utilizando-se microplacas de 96 poços, sendo que $20 \mu \mathrm{L}$ de cada solução inicial de extrato inicial foram transferidos para cada um dos poços, e em seguida, o volume final foi completado para $200 \mu \mathrm{L}$ de água destilada grau Milli-Q (Millipore). Desse modo, cada extrato foi diluído a $200 \mu \mathrm{g} / \mathrm{mL}$. Durante a realização do ensaio, $100 \mu \mathrm{L}$ do extrato diluído foram adicionados às suspensões de ovos de $H$. contortus, resultando em uma concentração final de extrato de $100 \mu \mathrm{g} / \mathrm{mL}$. Para a avaliação da atividade dos extratos vegetais contra as larvas de $H$. contortus, $30 \mu \mathrm{L}$ dos extratos vegetais diluídos à concentração de $2 \mathrm{mg} / \mathrm{mL}$ foram transferidos para as placas contendo $270 \mu \mathrm{L}$ de suspensão de larvas em meio de cultura, originando uma diluição final de $200 \mu \mathrm{g} / \mathrm{mL}$. Como controle negativo, 
foram utilizados DMSO 50\%, água destilada e como controle positivo, moxidectina dissolvida em DMSO 50\%, na concentração final de $100 \mu \mathrm{g} / \mathrm{mL}$.

Para a obtenção dos ovos de helmintos usados, empregou-se uma versão adaptada do teste de eclodibilidade, para determinação de resistência anti-helmíntica, proposto por World Association for the Advancement of Veterinary Parasitology - WAAVP (Coles et al., 1992). Foram utilizados ovinos criados em sistema semi-intensivo e com status parasitológico de contagem média de 2.000 OPG (ovos por grama de fezes), segundo o método de Gordon e Whitlock modificado (Ueno e Gutierrez, 1983). Aproximadamente $10 \mathrm{~g}$ de fezes, coletadas diretamente da ampola retal, foram acondicionadas em sacos plásticos e encaminhadas imediatamente ao laboratório, à temperatura ambiente. $\mathrm{O}$ material foi triturado em graal contendo $150 \mathrm{~mL}$ de solução salina hiper-saturada e foi filtrado em tamises de malhas de $250 \mathrm{mn} / \mu \mathrm{m}\left(\mathrm{n}^{\circ} 60\right)$ e $180 \mathrm{mn} / \mu \mathrm{m}\left(\mathrm{n}^{\circ}\right.$ 80). O material obtido foi acondicionado em tubos de centrífuga $(1,4 \times 9,8 \mathrm{~cm})$ e centrifugado a $2.000 \mathrm{rpm}$ por dois minutos. Transferiu-se o sobrenadante para outro tubo de centrífuga, repetindo-se três vezes o último procedimento para lavagens consecutivas com água destilada. $\mathrm{Na}$ última lavagem, o sedimento foi mantido com um pequeno volume de água destilada, ressuspendido e transferido para tubos de ensaio $(1,8 \times 10 \mathrm{~cm})$ em alíquotas de $200 \mu \mathrm{L}$, de modo a comporem uma suspensão com ovos de helmintos, contados com auxílio de microscópio óptico.

A análise do efeito dos extratos vegetais sobre o desenvolvimento de ovos de helmintos, através do teste de eclodibilidade (TE), foi realizada em placas de 96 poços, contendo $100 \mu \mathrm{L}$ da suspensão de ovos (equivalente a 100 ovos) por poço, em que se adicionou igual volume do tratamento (extrato vegetal) a ser estudado, preparado de modo que a concentração final resultante fosse $100 \mu \mathrm{g} / \mathrm{mL}$. As placas foram vedadas com filme plástico e incubadas em estufa à temperatura de $26 \pm 1^{\circ} \mathrm{C}$, por $48 \mathrm{~h}$, umidade relativa de $100 \%$. O tempo decorrido entre a coleta de fezes e o início da incubação dos ovos sob a ação dos extratos das plantas foi de, no máximo, duas horas. Avaliou-se a ação dos extratos vegetais sobre o desenvolvimento dos ovos, após o período de incubação de 48h, através de leitura em microscopia óptica, no aumento de 100 vezes. Foram avaliados todos os ovos presentes na amostra, de modo a classificálos de acordo com o estágio de desenvolvimento em que se encontravam. Cada tratamento foi feito em triplicata e os extratos que apresentaram atividade foram testados posteriormente no desenvolvimento larval.

O teste de desenvolvimento larval (TDL) foi proposto por Demeler et al. (2010). Realizou-se o ensaio em placa de 24 poços. Cada poço recebeu $200 \mu \mathrm{L}$ de água destilada, $20 \mu \mathrm{L}$ de suspensão contendo 100 ovos e $50 \mu \mathrm{L}$ de meio de crescimento, que foi preparado a partir de uma mistura na proporção de 2:2:1 de levedura:extrato de Earle, anfotericina B (Sigma A $2942,0.5 \mathrm{mg} / \mathrm{mL}$ ) e $1,5 \mathrm{mg} / \mathrm{mL}$ de Escherichia coli K12 liofilizada (suspensa em água destilada, autoclavada). Após 24 horas da eclosão dos ovos, foram adicionados $30 \mu \mathrm{L}$ de extrato vegetal, totalizando um volume de $300 \mu \mathrm{L}$. Incubou-se a placa a $27^{\circ} \mathrm{C}$ por 7 dias. Após esse período, as larvas viáveis foram contadas.

Os experimentos foram avaliados a partir da análise de variância do tipo ANOVA de uma entrada, seguida de comparações múltiplas entre médias pelo teste Tukey, considerando-se diferenças entre as médias significantes se $\mathrm{p}<0.05$ (GraphPad Prism 5.0).

\section{RESULTADOS}

A Tabela 1 relaciona os extratos vegetais que apresentaram atividade contra ovos de nematoides, fornece os dados botânicos das espécies usadas para obtenção desses extratos, assim como a parte do vegetal que foi usada. ANOVA de uma entrada realizada com os extratos orgânicos mostrou diferenças significantes $\quad\left(\mathrm{F}_{(79,160)}=5,939 ; \quad \mathrm{X}^{2}=0,7457\right.$; $\mathrm{p}<0,001)$ entre tratamentos e controles, assim como os extratos aquosos $\left(\mathrm{F}_{(74,150)}=4,163\right.$; $\left.\mathrm{X}^{2}=0,6725 ; \mathrm{p}<0,001\right)$. A figura $1 \mathrm{~A}$ mostra os resultados do processo de triagem realizado para os extratos orgânicos em comparação com os grupos controle e a Figura 1B mostra os resultados do processo de triagem realizado para os extratos aquosos em comparação com os grupos controle. Na Figura 1, estão representados os resultados mais significantes obtidos com extratos orgânicos e aquosos em comparação aos grupos controle. Dos extratos testados, 
sete orgânicos e seis aquosos apresentaram significância na capacidade de inibir a eclosão de ovos em relação ao controle positivo. Os extratos que interferiram com a eclosão dos ovos foram testados no teste de desenvolvimento larval
(TDL) e, baseado na ANOVA de uma entrada, nenhum extrato refletiu a eficácia anteriormente observada para os ovos $\quad\left(\mathrm{F}_{(11,12)}=11.47\right.$; $\left.X^{2}=0,9131 ; p<0,001\right)$ (Fig. 1A).

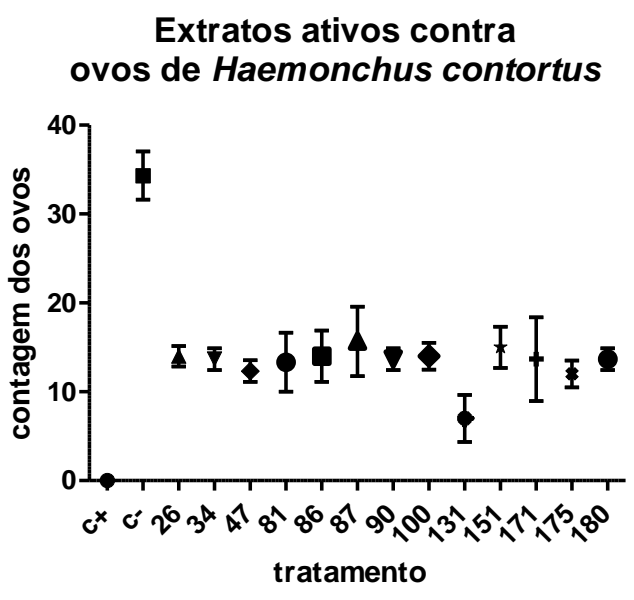

A

\section{Análise de extratos vegetais contra larvas de Haemonchus contortus}

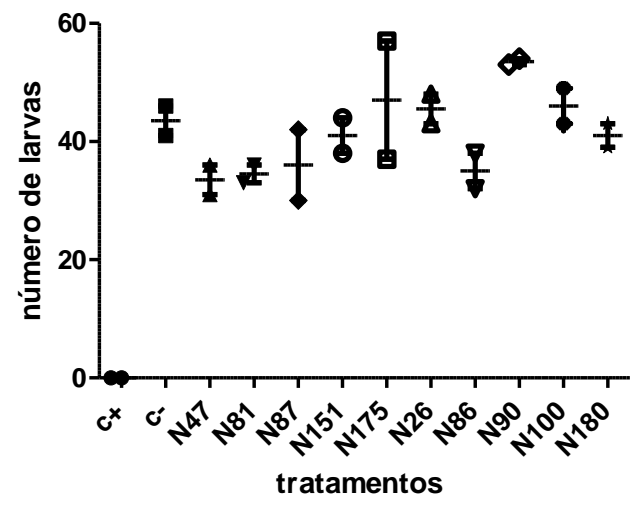

Figura 1. A) Resultados da triagem de extratos orgânicos obtidos de diferentes órgãos de plantas amazônicas contra ovos de Haemonchus contortus. B) Resultados da triagem de extratos aquosos obtidos de diferentes órgãos de plantas amazônicas contra ovos de Haemonchus contortus.

\section{DISCUSSÃO E CONCLUSÃO}

O método proposto permitiu a análise biológica de um número satisfatório de extratos vegetais e pode ser considerado como uma das formas mais rápidas e eficazes de se buscar produtos naturais bioativos. Essa metodologia é frequentemente usada na busca de extratos com atividade antimicrobiana, antifúngica e antitumoral (Younes et al., 2007), mas dados sobre a aplicação desse método na busca de plantas com atividade anti-helmíntica sobre nematódeos de ovinos são escassos.

Dos extratos vegetais testados, sete extratos orgânicos e seis aquosos apresentaram-se significantemente eficazes na inibição dos ovos de Haemonchus, na concentração de $100 \mu \mathrm{g} / \mathrm{mL}$. Segundo a literatura consultada, não existem dados publicados sobre a atividade anti-helmíntica dessas espécies. Moxidectina $(2 \mathrm{mg} / \mathrm{mL})$, controle positivo utilizado, inibiu $100 \%$ a eclosão dos ovos. Os extratos apresentaram desempenho promissor, quando comparado com a moxidectina. Nessa análise, é importante ressaltar que a moxidectina é um composto ativo isolado, enquanto os extratos são compostos por constituintes químicos de origem diversa, que podem agir de modo isolado ou em sinergismo, em relação à atividade ovicida/larvicida (Rates, 2001). No presente estudo, o extrato orgânico EB175, obtido de Trichilia sp., apresentou atividade ovicida significante. Os triterpenoides e limonoides isolados de algumas Meliaceae são descritos como potencialmente inseticidas contra Spodoptera frugiperda (lagarta do cartucho) (Sarria et al., 2011). Três extratos de plantas da família Euphorbiaceae inibiram a eclosão de ovos de modo significante, sendo dois aquosos, Mabea nitida (EB90) e Phyllanthus attenuatus (EB86), e um orgânico, Croton glandulosum (EB87). Às diversas substâncias isoladas de algumas plantas pertencentes a essa família são atribuídas propriedades antimicrobiana (Brusotti et al., 2011), antioxidante (Van Hong et al., 2011), antidiabética (QI et al., 2010), antitumoral (BALIGA et al., 2011) e anti-inflamatória (Okokon et al., 2010). O extrato orgânico de Tabernaemontana angulata (EB81), responsável 
por inibição significante dos ovos de Haemonchus, apresentou como compostos majoritários coronaridina e voacangina (Matheus de Assis et al., 2009). Marie-Magdalene et al. (2010) relatam a atividade anti-helmíntica de Tabernaemontana citrifolia sobre Haemonchus contortus. Suffredini et al. (2007b) descrevem os extratos orgânicos de Caryocar microcarpum (EB100) e Callophyllum brasiliense (EB47) como citotóxicos a células tumorais. A presença de cumarinas, xantonas, flavonoides e triterpenos em $C$. brasiliense (Clusiaceae) é descrita como responsável pela atividade citotóxica em várias linhagens de células (Cechinel Filho et al., 2009). No presente estudo, o extrato orgânico de C. microcarpum (Caryocaraceae) e o extrato aquoso de $C$. brasiliense inibiram de forma significante a eclosão de ovos de Haemonchus. O potencial anti-helmíntico de compostos isolados de espécies pertencentes à família Annonaceae já foi descrito em ensaios sobre nematódeos e protozoários, assim como foi descrita a presença de alcaloides e acetogeninas, compostos isolados frequentemente associados à atividade antiparasitária dessas plantas (Souza et al., 2008). No nosso estudo, o extrato orgânico de Guatteria riparia (EB151) foi responsável pela inibição significante da eclosão de ovos de $H$. contortus.

O extrato aquoso EB26, obtido de Aldina sp. e de uma espécie não identificada da família Myrtaceae (EB180) apresentaram atividade ovicida significante. Não existem menções de atividade biológica sobre nematódeos de pequenos ruminantes relacionadas a essas plantas na literatura. Porém, dados sobre atividades farmacológicas diversas ocorrem. A comparação da atividade relativa à inibição de eclosão de ovos desses extratos na concentração de $100 \mu \mathrm{g} / \mathrm{mL}$ com estudos semelhantes indica uma potencial atividade anti-helmíntica dessas plantas. Assis et al. (2003) relataram que o extrato acetato de etila de Spigelia anthelmia, responsável por $100 \%$ de atividade tóxica a ovos de $H$. contortus na concentração de $50 \mathrm{mg} / \mathrm{mL}$, inibiu em torno de $20 \%$ a eclosão de ovos na concentração de $3,1 \mathrm{mg} / \mathrm{mL}$.

No teste de desenvolvimento larval realizado com os extratos orgânicos e aquosos que apresentaram maior índice de inibição de eclosão de ovos, os resultados de inibição do desenvolvimento e morte de larvas demonstram ausência significante de atividade, quando comparados à substância de referência. De acordo com a literatura consultada, concentrações diversas dos extratos devem ser testadas. Costa et al. (2001), pesquisando a atividade anti-helmíntica de extratos brutos Azadirachta indica, apresentaram resultados de inibição de ovos de $H$. contortus de $98,22 \%$ com uma concentração de $3,12 \mathrm{mg} / \mathrm{mL}$ e $17,7 \%$ de inibição no desenvolvimento de larvas com o mesmo extrato e igual concentração. Quando o mesmo extrato foi empregado na concentração de $50 \mathrm{mg} / \mathrm{mL}$, a inibição no desenvolvimento das larvas aumentou para $87,1 \%$. É importante destacar que os testes realizados por esses autores mostram um efeito inibitório de larvas menor que o de eclodibilidade de ovos, quando se usa a mesma concentração de extrato. A inibição de $50 \%$ de eclosão de ovos foi observada na concentração de $0,36 \mathrm{mg} / \mathrm{mL}$, já a inibição no desenvolvimento de $50 \%$ de larvas, na concentração de $98 \mathrm{mg} / \mathrm{mL}$. Dados semelhantes também foram apresentados por AlShaibani et al. (2008). No presente estudo, os resultados de inibição de eclosão de ovos e de desenvolvimento larval são superiores aos encontrados na literatura, que descreve extratos vegetais ativos em concentrações mais elevadas dos que as utilizadas. Plantas que apresentaram ação letal aos ovos ou larvas no presente estudo necessitam de estudos mais aprofundados no que diz respeito à identificação dos componentes ativos presentes.

Com base nos resultados apresentados, os extratos vegetais selecionados oferecem uma oportunidade para a busca de novos compostos com atividade anti-helmíntica. No entanto, estudos mais detalhados são necessários para avaliar os componentes ativos e os mecanismos de ação dos extratos. Além disso, determinar uma curva dose-resposta, avaliação dos efeitos in vivo e toxicidade é necessário para validar seu uso como uma alternativa terapêutica e pode oferecer novas oportunidades de controle efetivo e econômico das verminoses em ovinos.

\section{AGRADECIMENTOS}

Agradecemos ao $\mathrm{CNPq}$ pela bolsa PIBIC de MPC, à Fapesp pelo auxílio 2008/58706-8, à CAPES e à Universidade Paulista pelo apoio. 


\section{REFERÊNCIAS}

AL-SHAIBANI, I.R.M.; PHULAN, M.S; ARIJOAND A.; QURESHI T.A. Ovicidal And Larvicidal Properties Of Adhatoda Vasica (L.) Extracts Against Gastrointestinal Nematodes Of Sheep in vitro. Pakistan Vet. J., v.28, p.79-83, 2008.

AMARANTE, A.F.T. Controle de endoparasitoses dos ovinos. In: MATOS, W.R.S. A produção animal na visão dos brasileiros. Piracicaba: FEALQ, 2001. p.461-473.

ASSIS, L.M.; BEVILAQUA, C.M.L.; MORAIS, S.M. et al. Ovicidal and larvicidal activity in vitro of Spigelia anthelmia Linn. extracts on Haemonchus contortus. Vet. Parasitol., v.117, p.43-49, 2003.

BALIGA, M.S; DSOUZA, J.J. Amla (Emblica officinalis Gaertn), a wonder berry in the treatment and prevention of cancer. Eur. J. Cancer Prev., v.20, p.225-39, 2011.

BRUSOTTI, G.; CESARI, I.; FRASSÀ, G. et al. Antimicrobial properties of stem bark extracts from Phyllanthus muellerianus (Kuntze) Excell. J. Ethnopharmacol., v.135, p.797-800, 2011.

CAPOBIANCO, J.P.R. Dossiê Mata Atlântica. Projeto monitoramento participativo da mata Atlântica. Instituto Socioambiental; Rede de ONGs Mata Atlântica, Sociedade Nordestina de Ecologia. São Paulo: Ipsis Gráfica e Editora. 2001. 407p.

CECHINEL FILHO, V.; MEYRE-SILVA, C.; NIERO, R. Chemical and pharmacological aspects of the genus Calophyllum. Chem. Biodivers., v.6, p.313-327, 2009.

COLES, G.C.; BAUER, F.H.M.; BORGSTEEDE, S. et al. World Association for the Advancement of Veterinary Parasitology (W.A.A.V.P.) Methods for detection of anthelmintic resistance in nematodes of veterinary importance. Vet. Parasitol., v.44, p.35-44, 1992.

DEMELER, J.; KÜTTLER, U.; EL-ABDELLATI, A et al. Standardization of the larval migration inhibition test for detection of resistance to ivermectin into gastro intestinal nematoids of ruminants. Vet Parasitol., v.174, p.58-64, 2010.
ECHEVARRIA, F.; PINHEIRO, A.C. Avaliação de resistência anti-helmíntica em rebanhos ovinos no município de Bagé, RS. Pesq. Vet. Bras., v.9, p.69-71, 1989.

EMBRAPA Caprinos e Ovinos, disponível em: <http://www.cnpc.embrapa.br/> acessado em 06/09/2012, às 14h30. Responsável pela página: Embrapa Caprinos e Ovinos, Sobral-CE.

GITHIORI, J.B.; ATHANASIADOU, S.; THAMSBORG, S.M. Use of plants in novel approaches for control of gastrointestinal helminthes in livestock with emphasis on small ruminants. Vet. Parasitol., v.139, p.308-320, 2006.

HAMANN, O. The Joint IUCN-WWF Plants conservation programme and its interest in medicinal plants. In: AKEREK, O.; HEYWOOD, V.; SYNGE, H. (eds.). The Conservation of Medicinal Plants. Cambridge: Cambridge University Press, 1991. p. 13-22.

JASMER, D.P.; McGUIRE, T.C. Antigens with application toward immune control of bloodfeeding parasitic nematodes. Br. Vet. J., v.152, p.251-268, 1996.

MARIE-MAGDELEINE, C.; MAHIEU, M.; D'ALEXIS, S. et al. In vitro effects of Tabernaemontana citrifolia extracts on Haemonchus contortus. Res. Vet. Sci., v.89, p.8892, 2010.

MATHEUS DE ASSIS, C.; MORENO, P.R.H.; YOUNG, M.C. et al. Isolamento e determinação da atividade biológica dos alcaloides majoritários de Tabernaemontana angulata. Rev. Bras. Farmacogn., v.19, p.626-631, 2009.

MITTERMEIER, R.A.; MYERS, N.; GIL, P.R.; MITTERMEIER, C.G. Hotspots. Cidade do México: Cemex S.A. 1999.

MOLENTO, M.B.; TASCA, C.; GALLO, A. et al. Método Famacha como parâmetro clínico individual de infecção por Haemonchus contortus em pequenos ruminantes. Cienc. Rural., v.34, p.1139-1145, 2004.

OKOKON, J.E.; NWAFOR, P.A. Antiinflammatory, analgesic and antipyretic activities of ethanolic root extract of Croton zambesicus. Pak. J. Pharm. Sci., v.23, p.385-392, 2010. 
PESSOA, L.M.; MORAIS, S.M.; BEVILÁCQUA, C.M.L. et al. Avaliação, in vitro, do efeito ovicida dos óleos essenciais de Chenopodium ambrosoides e Ocimum gratissimum sobre Haemonchus contortus. Cienc. Animal, v.11, p.63-65 2001.

QI, L.W.; LIU, E.H.; CHU, C. et al. Antidiabetic agents from natural products--an update from 2004 to 2009. Curr. Top. Med. Chem., v.10, p.434-57, 2010.

RAMOS, C.I.; BELLATO, V.; SOUZA, A.P. et al. Epidemiologia das helmintoses gastrintestinais de ovinos no Planalto Catarinense. Cienc. Rural, v.34, p.1889-1895, 2004.

RATES, S.M.K. Plants as source of drugs. J. Int. Soc. Toxinol., v.39, p.603-13, 2001.

SARRIA, A.L.; SOARES, M.S.; MATOS, A.P. et al. Effect of triterpenoids and limonoids isolated from Cabralea canjerana and Carapa guianensis (Meliaceae) against Spodoptera frugiperda (J.E. SMITH). Z Naturforsch C., v.66, p.245-50, 2011.

SOUZA, M.M.C.; BEVILACQUA, C.M.L.; MORAIS, S.M. et al. Anthelminthic acetogenin from Annosa aquamosa L. Seeds. An. Acad. Bras. Cienc., v.80, p.271-277, 2008.
SUFFREDINI, I.B.; VARELLA, A.D.; YOUNES, R.N. Cytotoxic molecules from natural sources. Tapping the Brazilian biodiversity. Anti-Canc. Agents Med. Chem., v.6, p.367-75, 2006.

SUFFREDINI, I.B.; PACIENCIA, M.L.B.; FRANA, S.A. et al. In vitro breast cancer cell lethality by Brazilian plant extracts. Pharmazie, v.62, p.798-800, 2007a.

SUFFREDINI, I.B.; PACIENCIA, M.L.; VARELLA, A.D.; YOUNES, R.N. In vitro cytotoxic activity of Brazilian plant extracts against human lung, colon and CNS solid cancers and leukemia. Fitoterapia, v.78, p.223-6, 2007b.

UENO, H.; GUTIERREZ, V.C. Manual para o diagnóstico das helmintoses de ruminantes. Japan International Cooperation Agency, 1983. $176 \mathrm{p}$.

VAN HONG, N.T.; RIVIÈRE, C.; HONG, Q.T. et al. Identification by LC-ESI-MS of flavonoids responsible for the antioxidant properties of Mallotus species from Vietnam. Nat. Prod. Commun., v.6, p.813-8, 2011.

YOUNES, R.N.; VARELLA, A.D.; SUFFREDINI, I.B. Discovery of new antitumoral and antibacterial drugs from Brazilian plant extracts using high throughput screening. Clinics. v.62, p.763-8, 2007b. 